\title{
Two dimensional electrical conductivity model of the solid state plasma for SPiN device
}

\author{
Haiyan Kang ${ }^{1 \mathrm{a})}$, Huiyong $\mathrm{Hu}^{1}$, Bin Wang ${ }^{1}$, Han $\mathrm{Su}^{1}$, Minru Hao ${ }^{1}$, \\ and Yingbo $\mathbf{Z h a o}^{2}$ \\ ${ }^{1}$ School of Microelectronics, Xidian University, \\ 2 South Taibai Road, Xi'an 710071, P. R. China \\ ${ }^{2} X i$ 'an University of Architecture and Technology, \\ 13 Yanta Road, Xi'an 710055, P. R. China
}

a)kanghaiyan5200@163.com

\begin{abstract}
The paper proposed a two dimensional (2D) electrical conductivity model based on the solid state plasma concentration distribution model. The results indicated that the imaginary part of the electrical conductivity is more sensitive to the electromagnetic signal frequency than the real part. And the real part is mainly effected by the internal properties of the solid state plasma. Finally, a smart solid state plasma dipole antenna is designed to test the effect of the imaginary part of the electrical conductivity on the radiation characteristics of the antenna. Plasma dipole antenna has also been compared with metal dipole antenna, the results show that the plasma antenna is entirely possible to replace the metal antenna.
\end{abstract}

Keywords: solid state plasma, electrical conductivity, SPiN

Classification: Electron devices, circuits and modules

\section{References}

[1] A. E. Fathy, et al.: "Silicon-based reconfigurable antennas-concepts, analysis, implementation, and feasibility," IEEE Trans. Microw. Theory Techn. 51 (2003) 1650 (DOI: 10.1109/TMTT.2003.812559).

[2] M. ElSherbiny, et al.: "Holographic antenna concept, analysis, and parameters," IEEE Trans. Antennas Propag. 52 (2004) 830 (DOI: 10.1109/TAP.2004. 824673).

[3] Y. Yashchyshyn, et al: "Development and investigation of an antenna system with reconfigurable aperture," IEEE Trans. Antennas Propag. 57 (2009) 2 (DOI: 10.1109/TAP.2008.2009728).

[4] P. Cova, et al:: "A software tool for the design of high power PiN diodes based on the numerical study of the reverse characteristics," Solid-State Electron. 63 (2011) 60 (DOI: 10.1016/j.sse.2011.05.009).

[5] Y. Aydogdu, et al:: "Solid state electrical conductivity properties of copper complexes of novel oxime compounds oxolane ring," Mater. Lett. 57 (2003) 3755 (DOI: 10.1016/S0167-577X(03)00174-5). 
[7] R. P. Jackson, et al.: "Physical modeling of millimetre wave signal reflection from forward biased PIN diodes," Solid-State Electron. 54 (2010) 149 (DOI: 10.1016/j.sse.2009.12.009).

[8] Y. C. Zhai, et al:: "Simulation and structure analysis of reconfigurable solid plasma channel based on SPINs," Microelectron. Eng. 145 (2015) 49 (DOI: 10. 1016/j.mee.2015.03.003).

[9] B. B. Jayant: Fundamentals of Power Semiconductor Devices (Springer Science, Carolina, 2008) 204.

[10] A. Afzalian, et al:: "Physical modeling and design of thin-film SOI lateral PIN photo diodes," IEEE Trans. Electron Devices 52 (2005) 1116 (DOI: 10.1109/ TED.2005.848080).

[11] A. Nussbaum: "The modified fletcher boundary conditions," Solid-State Electron. 18 (1975) 107 (DOI: 10.1016/0038-1101(75)90078-7).

[12] R. T. Kinasewitz and B. Senitzky: "Investigation of the complex permittivity of n-type silicon at millimeter wavelengths," J. Appl. Phys. 54 (1983) 3394 (DOI: 10.1063/1.332452).

\section{Introduction}

Silicon-based solid state plasma antenna based on silicon surface PiN (SPiN) device is characterized by many unique advantages, which will have a very broad application prospects in the future wireless communication $[1,2,3]$. As a basic radiation unit, SPiN device plays an important role in the solid state plasma antenna. In such a semiconductor device, when activated, the solid state plasma with high concentration is formed in the intrinsic region (I-region), so the plasma will appear to be of metal-like characteristic due to fairly high electrical conductivity $[4,5]$.

The electrical conductivity as a crucial parameter in the study of plasma transport properties is of fundamental importance to the design of the plasma antenna. So far, in the antenna's simulation, the two-dimensional distribution of the electrical conductivity within the I-region is considered as uniform [6, 7], but the electrical conductivity as a function of plasma concentration must be nonuniform. Moreover, the electrical conductivity will turn into a complex under alternating electric field when an electromagnetic signal is incident on the solid state plasma and which will affect the radiation characteristics of the antenna [8]. However, the reports on the imaginary part of the electrical conductivity have been rare.

In this paper, an effective model for electrical conductivity based on the $2 \mathrm{D}$ solid state plasma concentration distribution model and Drude's model is developed. The variation trend of the real and imaginary part of the electrical conductivity with the parameters such as average solid state plasma concentration, electromagnetic signal frequency and relaxation time have been discussed. And the effect of the imaginary part on the radiation characteristics of the antenna has been tested. 


\section{Two dimensional model of the electrical conductivity}

\subsection{Two dimensional concentration distribution model of solid state plasma}

Under steady-state conditions, the solid state plasma concentration will form a steady distribution in the I-region. Let us consider the length direction is X-axis and the depth direction is Y-axis, as shown in Fig. 1. The concentration distribution of the plasma in the SPiN along X-axis is presented in Fig. 2. In order to simplify the model and highlight its physical meaning, it is essential to make the following assumptions:

(1) The SPiN devices works at the high-level injection condition;

(2) Quasi neutral condition $(n(x, y)=p(x, y))$ at each point is valid within the I-region;

(3) Supposing that the transport of the carriers diffuse along the X-axis firstly, then, setting the $\mathrm{X}$-axis as a starting point, diffuse along the Y-axis.

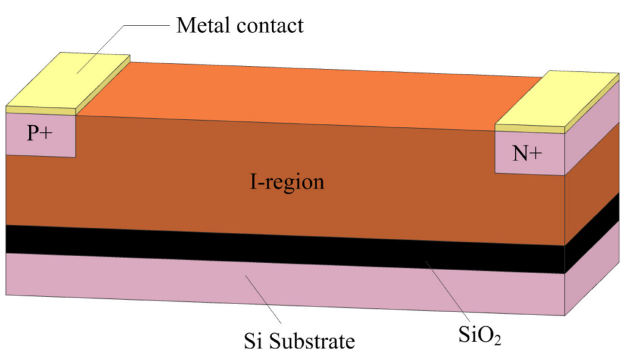

Fig. 1. Structure of the SPiN device.

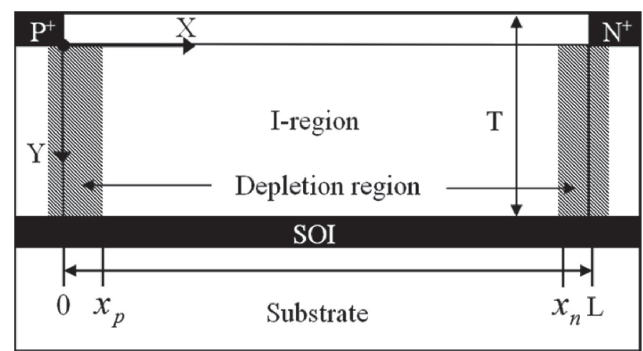

Fig. 2. Cross-sectional view of the SPiN device.

At the high-injection condition, when the 'plasma' condition exists within the I-region, the excess solid state plasma concentration exceeds the base doping level by several orders of magnitude, the assumption (3) is valid. The electrons and holes distributions within the I-region can be obtained by solving the continuity equation [9]:

$$
\begin{aligned}
& \frac{\partial n(x, y)}{\partial \tau}=-\frac{n(x, y)}{\tau}+D_{n} \frac{\partial^{2} n(x, y)}{\partial x \partial y}+\mu_{n} \frac{\partial}{\partial x}(n E) \\
& \frac{\partial p(x, y)}{\partial \tau}=-\frac{p(x, y)}{\tau}+D_{p} \frac{\partial^{2} p(x, y)}{\partial x \partial y}-\mu_{p} \frac{\partial}{\partial x}(p E)
\end{aligned}
$$

where $D_{n}$ and $D_{p}$ are diffusion coefficients for electrons and holes, respectively, and $\tau$ is the high level lifetime within the I-region. Combining the Eq. (1a) and Eq. (1b) 
after multiplying (1a) by $\left(\mu_{p} p\right)$ and (1b) by $\left(\mu_{n} n\right)$. And that the transport of the carriers due to the electric field can be neglected. Since the plasma concentration in accordance with the quasi neutral condition $n(x, y)=p(x, y)$ can be written under steady state conditions as:

$$
\frac{\partial^{2} n(x, y)}{\partial^{2} \tau}=0=-\frac{n(x, y)}{\tau}+D_{a} \frac{\partial^{2} n(x, y)}{\partial x \partial y} \quad(0 \leq x \leq L, 0 \leq y \leq T)
$$

where $D_{a}$ is the ambipolar diffusion coefficient given by $D_{a}=2 D_{n} D_{p} /\left(D_{n}+D_{p}\right)$. $\mathrm{L}$ and $\mathrm{T}$ are the I-region length and the depth, respectively.

In steady-state forward bias operation, at a given depth $\mathrm{y}$, the plasma concentration along the $\mathrm{X}$-axis has a distribution of catenary form with just two exponential basis functions [10]. However, at a given $\mathrm{x}$, the plasma concentration presents an exponential decay along the Y-axis. General solutions are shown in Eq. (3):

$$
\left\{\begin{array}{l}
n(x)=A \exp \left(\frac{x}{L_{a}}\right)+B \exp \left(-\frac{x}{L_{a}}\right) \\
n(y)=C \exp \left(-\frac{y}{L_{a}}\right)
\end{array} \quad(0 \leq x \leq L, 0 \leq y \leq T)\right.
$$

where $L_{a}$ is the diffusion length given by $L_{a}=\sqrt{D_{a} \tau}, \mathrm{A}, \mathrm{B}$ and $\mathrm{C}$ are arbitrary constants determined by boundary conditions at the $\mathrm{Pi}$, Ni junctions and the surface concentration of the SPiN device.

The Fletcher boundary conditions which can be used in any junction [11] are used to determine the coefficients A and B. It is worth stressing that the Fletcher boundary condition is more accurate than the Shockley boundary condition at the high-level injection.

The equilibrium concentration of plasma at the interface $x=x_{p}$ is shown by Fletcher boundary condition to be:

$$
n\left(x_{p}\right)=\frac{\left(m_{o p}+m_{o i} \gamma_{1}\right) \gamma_{1}}{1-\gamma_{1}^{2}}
$$

where

$$
\gamma_{1}=\exp \left[q\left(V_{P i}-V_{D 1}\right) / k T\right]
$$

where $m_{o p}$ and $m_{o i}$ are equilibrium majority plasma concentration in the $\mathrm{P}^{+}$region and I-region. $V_{P i}$ and $V_{D 1}$ is the applied forward bias voltage drop and the barrier potential of the junction, respectively.

We assume that all impurities are ionized completely, then $m_{o p}$ and $m_{o i}$ can be simplified as:

$$
m_{o p}=N_{A}-\frac{n_{i e f f}^{2}}{N_{A}}, \quad m_{o i}=N_{d}-\frac{n_{i}^{2}}{N_{d}}
$$

However,

$$
N_{A} \gg \frac{n_{i e f f}^{2}}{N_{A}}, \quad N_{d} \gg \frac{n_{i}^{2}}{N_{d}}, \quad N_{A} \gg N_{d}, \quad \gamma_{1}<1
$$

Thus, $n\left(x_{\mathrm{p}}\right)$ is given as: 


$$
n\left(x_{p}\right)=\frac{\gamma_{1}\left[N_{A}-\frac{n_{i e f f}^{2}}{N_{A}}+\left(N_{d}-\frac{n_{i}^{2}}{N_{d}}\right) \gamma_{1}\right]}{1-\gamma_{1}^{2}} \approx \gamma_{1} N_{A}
$$

where $n_{\text {ieff }}$ is the effective intrinsic carrier concentration of the $\mathrm{P}^{+}$region under the consideration of the narrowing effect of the bandgap, $n_{i}$ is the intrinsic carrier concentration of the I-region. $N_{A}, N_{d}$ and $N_{D}$ denote doping concentration of $\mathrm{P}^{+}$ region, I-region and $\mathrm{N}^{+}$region, respectively.

Similarly, the plasma concentration at the interface $x=x_{n}$ by the Fletcher can be simplified as:

$$
n\left(x_{n}\right) \approx \gamma_{2} N_{D}
$$

where $\gamma_{2}=\exp \left(q\left(V_{N i}-V_{D 2}\right) / k T\right), V_{N i}$ and $V_{D 2}$ is the applied forward bias voltage drop and barrier potential of the $N i$ junction, respectively.

According to the assumption (3), the boundary conditions for Eq. (3) can be written as

$$
\left.n(y)\right|_{y=0}=n(x)
$$

Substituting the boundary conditions Eq. (8), Eq. (9) and Eq. (10) into the Eq. (3), the coefficients $\mathrm{A}, \mathrm{B}$ and $\mathrm{C}$ can be obtained as:

$$
\begin{gathered}
A=\frac{\gamma_{1} N_{A} \exp \left(-\frac{x_{n}}{L_{a}}\right)-\gamma_{2} N_{D} \exp \left(-\frac{x_{p}}{L_{a}}\right)}{\exp \left(-\frac{x_{n}-x_{p}}{L_{a}}\right)-\exp \left(\frac{x_{n}-x_{p}}{L_{a}}\right)} \\
B=\frac{\gamma_{1} N_{A} \exp \left(\frac{x_{n}}{L_{a}}\right)-\gamma_{2} N_{D} \exp \left(\frac{x_{p}}{L_{a}}\right)}{\exp \left(\frac{x_{n}-x_{p}}{L_{a}}\right)-\exp \left(-\frac{x_{n}-x_{p}}{L_{a}}\right)} \\
C=A \exp \left(\frac{x}{L_{a}}\right)+B \exp \left(-\frac{x}{L_{a}}\right)
\end{gathered}
$$

where $x_{p}=\sqrt{\frac{2 \varepsilon_{S i} \varepsilon_{0} V_{D 1}}{q N_{d}}}$ and $x_{n}=L-\sqrt{\frac{2 \varepsilon_{S i} \varepsilon_{0} V_{D 2}}{q N_{d}}}$.

Therefore, the $2 \mathrm{D}$ concentration distribution model of the solid state plasma within the I-region can be written as:

$$
\begin{aligned}
n(x, y)= & \frac{N_{A} \exp \left[\frac{q\left(V_{P i}-V_{D 1}\right)}{k T}-\frac{x_{n}}{L_{a}}\right]-N_{D} \exp \left[\frac{q\left(V_{N i}-V_{D 2}\right)}{k T}-\frac{x_{p}}{L_{a}}\right]}{\exp \left(-\frac{x_{n}-x_{p}}{L_{a}}\right)-\exp \left(\frac{x_{n}-x_{p}}{L_{a}}\right)} \exp \left(\frac{x-y}{L_{a}}\right) \\
+ & \frac{N_{A} \exp \left[\frac{q\left(V_{P i}-V_{D 1}\right)}{k T}+\frac{x_{n}}{L_{a}}\right]-N_{D} \exp \left[\frac{q\left(V_{N i}-V_{D 2}\right)}{k T}+\frac{x_{p}}{L_{a}}\right]}{\exp \left(\frac{x_{n}-x_{p}}{L_{a}}\right)-\exp \left(-\frac{x_{n}-x_{p}}{L_{a}}\right)} \exp \left(-\frac{x+y}{L_{a}}\right)
\end{aligned}
$$

$(0 \leq x \leq L, 0 \leq y \leq T)$ 


$$
\left\{\begin{array}{l}
V_{P i}=\frac{1}{2}\left[V+\frac{k T}{q} \ln \left(\frac{\alpha}{\beta}\right)\right] \\
V_{N i}=\frac{1}{2}\left[V-\frac{k T}{q} \ln \left(\frac{\alpha}{\beta}\right)\right]
\end{array}\right.
$$

where

$$
\begin{aligned}
& \alpha=\frac{N_{D} D_{n} \exp \left(\frac{x_{n}-x_{p}}{L_{a}}\right)+D_{n} N_{D} \exp \left[\frac{x_{p}-x_{n}}{L_{a}}\right]-2 N_{D} D_{p}}{2 N_{A} D_{n}-N_{A} D_{p} \exp \left[\frac{x_{p}-x_{n}}{L_{a}}\right]-N_{A} D_{p} \exp \left(\frac{x_{n}-x_{p}}{L_{a}}\right)} \\
& \beta=\exp \left[q\left(V_{D 2}-V_{D 1}\right) / k T\right]
\end{aligned}
$$

\subsection{Two dimensional electrical conductivity model of the solid state plasma}

Electrical conductivity is a critical parameter in the research on the radiation character of the silicon-based solid state plasma antenna. The influence of conduction band charge carriers on the propagation of electromagnetic waves is characterized by a complex electrical conductivity $\sigma$, where the real part $\left(\sigma_{\mathrm{r}}\right)$ is the displacement current density and the imaginary part $\left(\sigma_{\mathrm{i}}\right)$ is the conduction current density.

$$
\sigma=\sigma_{r}-j \sigma_{i}
$$

At the high-level injection, the solid state plasma is dictated by the presence of both electrons and holes in the I-region, consequently, the electrical conductivity is calculated using the relationship [12]

$$
\sigma(x, y)=\frac{n(x, y) e^{2}}{m_{c n}^{*}} \frac{t}{1-j \omega t}+\frac{n(x, y) e^{2}}{m_{c p}^{*}} \frac{t}{1-j \omega t}
$$

where $t$ is the relaxation time, $\omega$ is electromagnetic signal angular frequency, $m_{c n}^{*}$ and $m_{c p}^{*}$ is the conductivity effective mass of the electrons and holes, respectively. Hence, the real and imaginary parts of electrical conductivity can be written as

$$
\begin{aligned}
& \sigma_{r}(x, y)=\frac{n(x, y) e^{2} t}{1+\omega^{2} t^{2}}\left(\frac{1}{m_{c n}^{*}}+\frac{1}{m_{c p}^{*}}\right) \\
& \sigma_{i}(x, y)=\frac{n(x, y) e^{2} \omega t^{2}}{1+\omega^{2} t^{2}}\left(\frac{1}{m_{c n}^{*}}+\frac{1}{m_{c p}^{*}}\right)
\end{aligned}
$$

where

$$
\begin{aligned}
m_{c n}^{*} & =\left(1 / 3 m_{l}+2 / 3 m_{t}\right)^{-1}, m_{c p}^{*}=\left(m_{p h}^{3 / 2}+m_{p l}^{3 / 2}\right) /\left(m_{p h}^{1 / 2}+m_{p l}^{1 / 2}\right), \\
m & =m_{c} m_{p} /\left(m_{c}+m_{p}\right) .
\end{aligned}
$$

$m_{l}$ and $m_{t}$ is the longitudinal and transverse effective mass of conduction band electrons in silicon, we will use the known values $m_{l}=0.98 m_{0}$ and $m_{t}=0.19 m_{0}$, where $m_{0}$ is the rest mass of the free electron. $m_{p h}$ and $m_{p l}$ is the effective mass of the heavy hole and light hole. 
The relative permittivity of the solid state plasma is also a complex, which can be expressed by the real and imaginary part of electrical conductivity,

$$
\varepsilon=\varepsilon_{r}-j \varepsilon_{i}=\varepsilon_{s}-\frac{\sigma_{r} t}{\varepsilon_{0}}-j \frac{\sigma_{i}}{\omega^{2} \varepsilon_{0} t}
$$

where $\varepsilon_{S}$ is the contribution to the relative dielectric constant of the bound electrons (for silicon, $\varepsilon_{s}=11.7$ ). Therefore, the loss tangent is given by

$$
\tan \delta=\frac{\varepsilon_{i}}{\varepsilon_{r}}=\frac{\sigma_{i}}{\left(\varepsilon_{s} \varepsilon_{0} t-\sigma_{r} t^{2}\right) \omega^{2}}
$$

The loss $\operatorname{tangent} \tan \delta$ is a function of imaginary parts of electrical conductivity and electromagnetic signal angular frequency when the average solid state plasma concentration is considered to be a constant. Because that the real part of relative permittivity and electrical conductivity is mainly affected by the inner properties of the solid state plasma.

\section{Results and discussion}

\subsection{The simulation of the solid state plasma concentration model}

The 2D solid state plasma concentration distribution model is validated by comparing the model prediction of different sets of parameters with the TCAD simulation results. In the simulation, the bandgap narrow model and recombination model are used. Regarding mobility model, we have used carrier-carrier scattering and doping dependent model with high field saturation models. All simulations assumed at a temperature of $300 \mathrm{~K}$.

The solid state plasma concentration distribution model is first tested on a SPiN with $\mathrm{L}=80 \mathrm{um}, \mathrm{T}=80 \mathrm{um}, \mathrm{N}_{\mathrm{A}}=\mathrm{N}_{\mathrm{D}}=2 \mathrm{e} 19 \mathrm{~cm}^{-3}, \mathrm{~N}_{\mathrm{d}}=1 \mathrm{e} 13 \mathrm{~cm}^{-3}$ under an applied voltage $\mathrm{V}=1 \mathrm{~V}$.
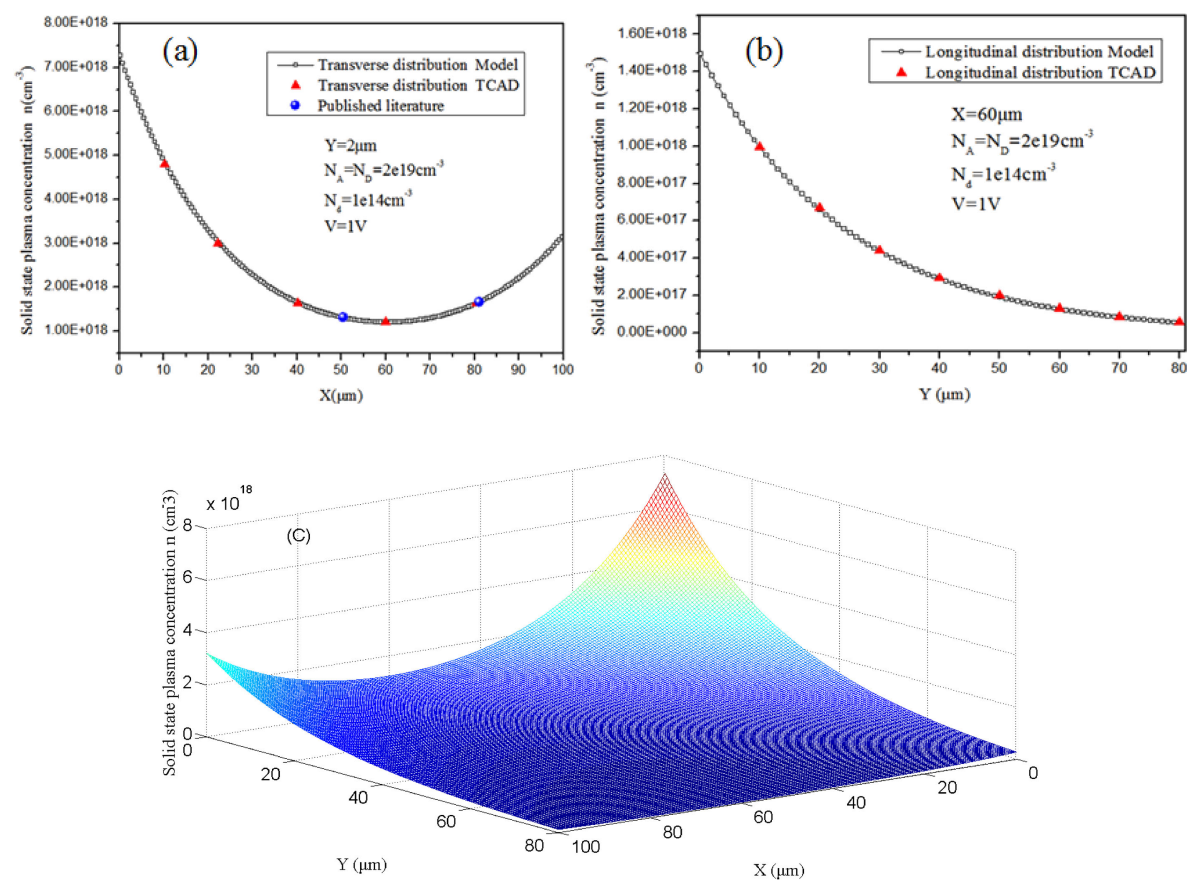

Fig. 3. Concentration distribution of the solid state plasma: (a) transverse distribution (b) Longitudinal distribution (c) Two dimensional distribution 
At a given depth $\mathrm{Y}=2 \mathrm{um}$, the transverse (X-axis direction) plasma distribution was illustrated in Fig. 3(a), the largest holes and electrons concentration in the I-region occur at its boundaries. The lowest plasma concentration is not located the center of the $\mathrm{X}$-axis due to the mobility difference of the electrons and holes. However, at a given point $\mathrm{X}=60 \mathrm{um}$, the transport of the plasma along the Y-axis presents exponential decay, which is shown in Fig. 3(b). The largest plasma concentration occurs at the surface of the I-region. Fig. 3(c) presents the 2D concentration distribution of the solid state plasma within the I-region. The comparison of the model and TCAD simulation result is presented and good agreements are clearly observed. Furthermore, the results also coincide with the information published [8].

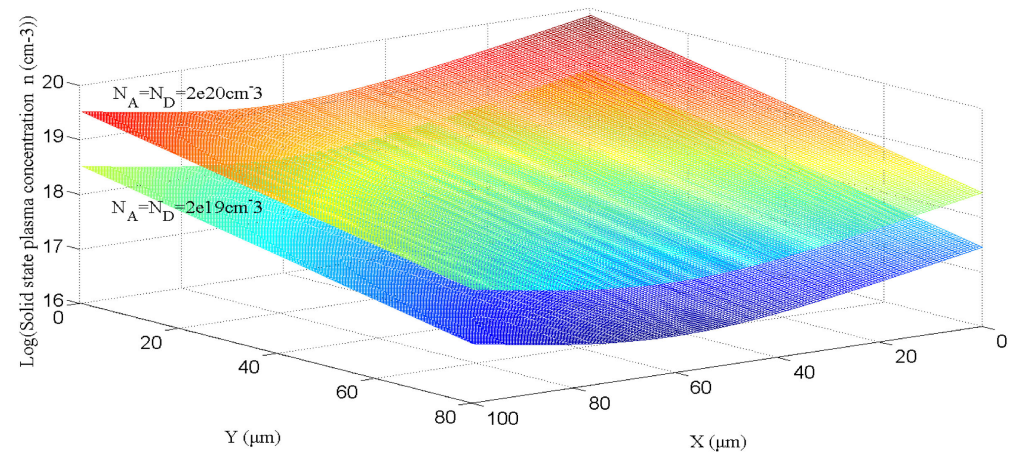

Fig. 4. Two dimensional plasma concentration distribution for different $\mathrm{P}^{+}$and $\mathrm{N}^{+}$doping level

Fig. 4 illustrates the logarithmic 2D concentration distribution of the solid state plasma with the different doping level of the $\mathrm{P}^{+}$and $\mathrm{N}^{+}$region. It is characterized by I-region length $\mathrm{L}=100 \mathrm{um}$ and the depth $\mathrm{T}=80 \mathrm{um}$. In general, we assume that the doping level of $\mathrm{P}^{+}$and $\mathrm{N}^{+}$regions is equal. Note the significant reduction in the solid state plasma concentration if the doping level reduces an order of magnitude.
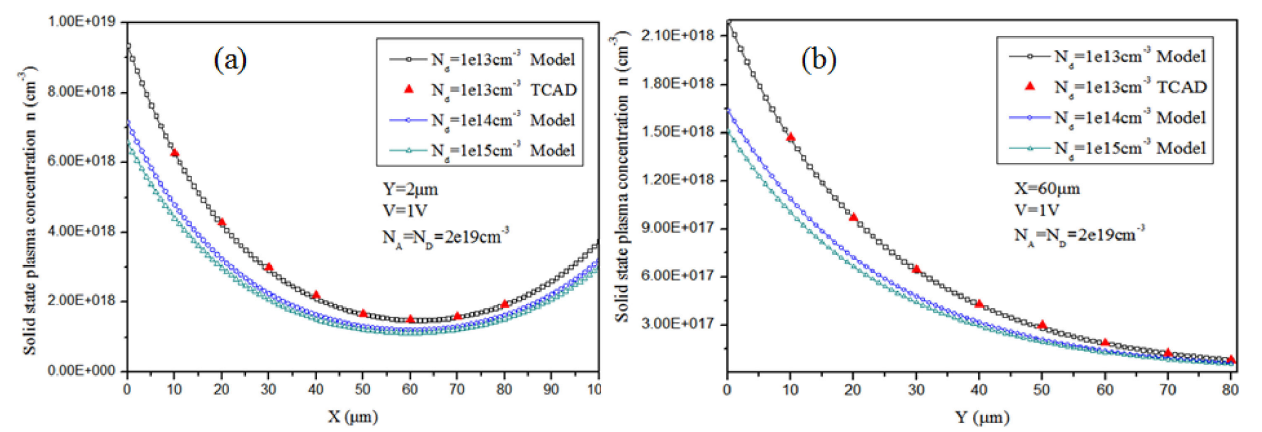

Fig. 5. Concentration distribution of the solid state plasma for different $\mathrm{N}_{\mathrm{d}}$ : (a) transverse distribution (b) Longitudinal distribution 
the solid state plasma concentration will decrease when the doping level of the I-region increase. That is because the plasma density is proportional to the minority carrier concentration within the I-region, the higher the I-region doping, the lower the minority concentration and the plasma density. Good agreements between the model predication and TCAD results are obtained.

\subsection{The simulation of the electrical conductivity model}

To assess the two dimensional electrical conductivity distribution and the trend of the electrical conductivity with the average solid state plasma concentration (n), electromagnetic signal frequency (f) and the relaxation time (t), some MATLAB scripts were written using the proposed electrical conductivity model.

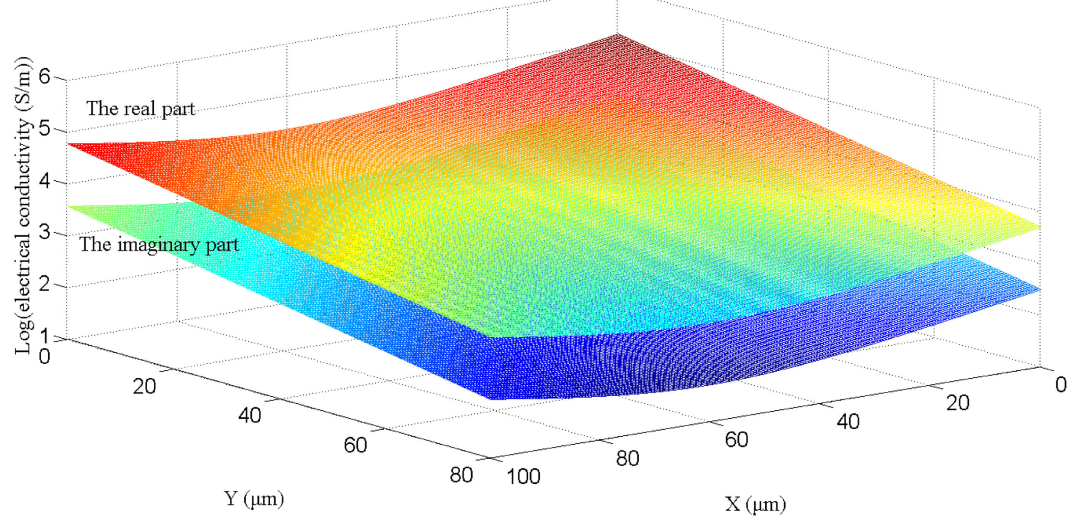

Fig. 6. Two dimensional electrical conductivity distribution

The real part and the imaginary part of the electrical conductivity distribution within the I-region at $\mathrm{f}=10^{11} \mathrm{~Hz}$ and $\mathrm{t}=10^{-13} \mathrm{~s}$ is shown in Fig. 6. It is clear to note that both the real and the imaginary part of the electrical conductivity show the same regularities of distribution, just the value of the real part is greater than that of the imaginary part. Moreover, due to a linear relationship between the electrical conductivity and concentration at a given $\mathrm{f}$ and $\mathrm{t}$, the $2 \mathrm{D}$ distribution of the electrical conductivity both on the real and imaginary parts are similar to the solid state plasma concentration distribution within the I-region.
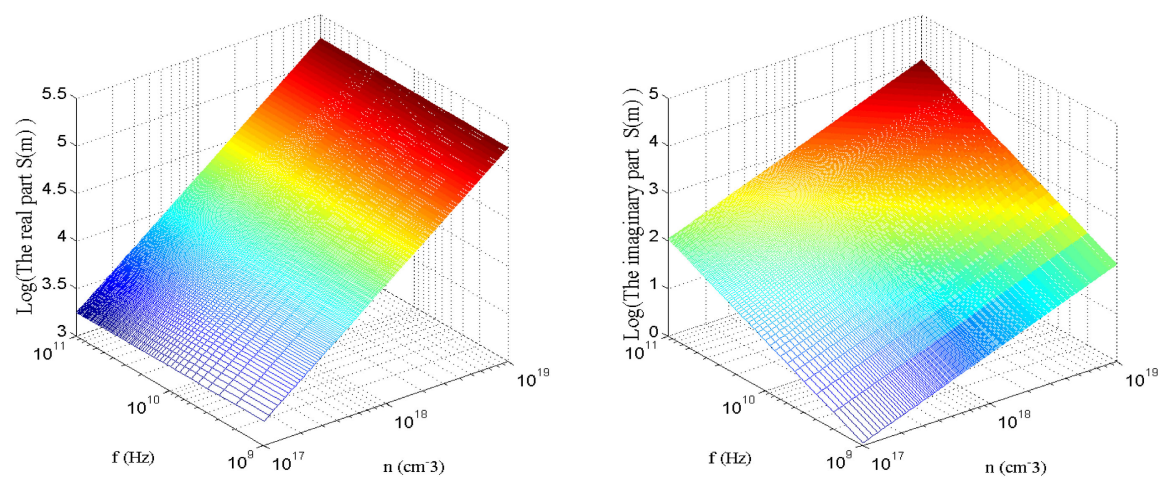

Fig. 7. Variation trend of the electrical conductivity with $\mathrm{n}$ and $\mathrm{f}$ 
The variation trend of the real and imaginary part of the electrical conductivity with the average plasma concentration (n) and electromagnetic signal frequency (f) is shown in Fig. 7 at a given relaxation time $t=10^{-13} \mathrm{~s}$. It is shown that the real and imaginary part of electrical conductivity both increase with the increasing average plasma concentration. But the real part keeps constant, when the imaginary part increases with the increasing electromagnetic signal frequency. Therefore, we can conclude that changes of electromagnetic signal frequency mainly affect the imaginary part of the electrical conductivity, but which has little effect on the real part.
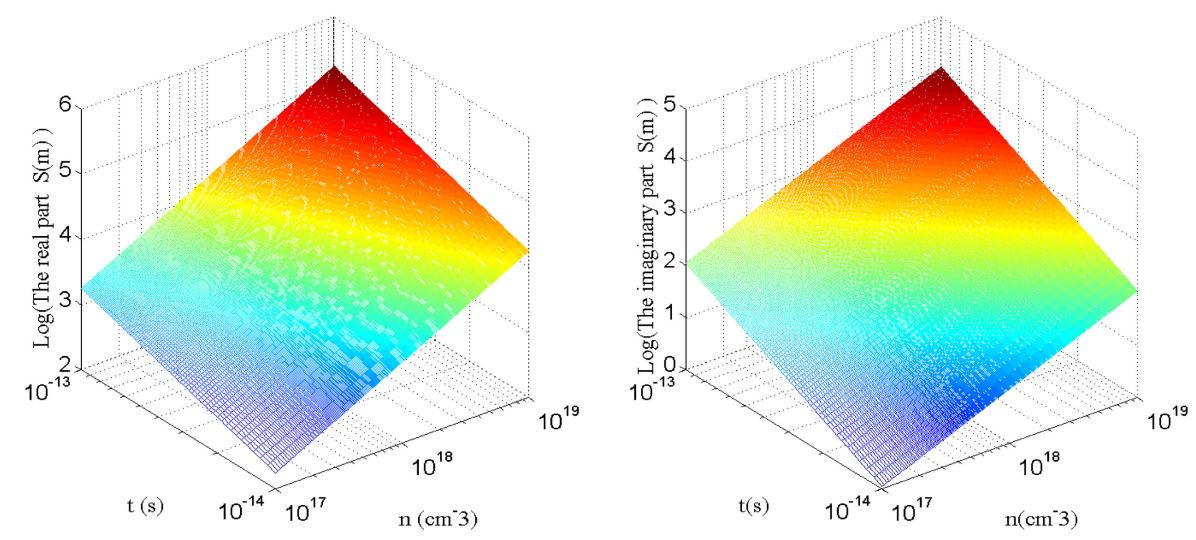

Fig. 8. Variation trend of the electrical conductivity with $n$ and $t$

The variation trend of the real and imaginary part of the electrical conductivity with the average solid state plasma concentration (n) and relaxation time $(\mathrm{t})$ is shown in Fig. 8 at a given electromagnetic signal frequency $\mathrm{f}=10^{11} \mathrm{~Hz}$. The results show that both the real and imaginary part of the electrical conductivity increases with the increasing of the plasma concentration, but decrease with the increasing of the relaxation time.

\subsection{The effect of the imaginary part of the electrical conductivity on the antenna}

Based on the simulation results of the model above, we can conclude that the real part of the electrical conductivity is mainly affected by the internal properties of the solid state plasma, such as concentration and relaxation time, but the imaginary part of the electrical conductivity is influenced by both the internal properties and the electromagnetic signal frequency. Therefore, we can verify the imaginary part of the electrical conductivity has an effect on the radiation characteristics of the antenna by the loss tangent $\tan \delta$ which is obtained through changing the electromagnetic signal frequency.

A smart silicon-based solid state plasma dipole and reference metal dipole were fabricated, and the average solid state plasma concentration in the I-region for the SPiN device is $1 \times 10^{18} \mathrm{~cm}^{-3}$, so the real part of the electrical conductivity and relative permittivity is $\sigma_{\mathrm{r}}=29717 \mathrm{~S} / \mathrm{m}$ and $\varepsilon_{\mathrm{r}}=-520$, respectively. The variation of the imaginary part and loss tangent $\tan \delta$ versus electromagnetic signal frequency is shown in Table I. And the characteristics of the antenna are plotted in Fig. 9. 
Table I. Variation of the imaginary part and $\tan \delta$ with electromagnetic signal frequency

\begin{tabular}{llllllllll}
\hline $\mathrm{f}$ & $5 \times 10^{10}$ & $6 \times 10^{10}$ & $7 \times 10^{10}$ & $8 \times 10^{10}$ & $9 \times 10^{10}$ & $1 \times 10^{11}$ & $2 \times 10^{11}$ & $3 \times 10^{11}$ & $5 \times 10^{11}$ \\
\hline$\sigma_{\mathrm{i}}$ & 235.5 & 282.6 & 329.7 & 376.8 & 423.8 & 470.9 & 941.2 & 1410.1 & 2340.8 \\
\hline $\tan \delta$ & 129 & 175 & 92.2 & 80.6 & 71.7 & 64.5 & 32.3 & 21.5 & 12.9 \\
\hline
\end{tabular}
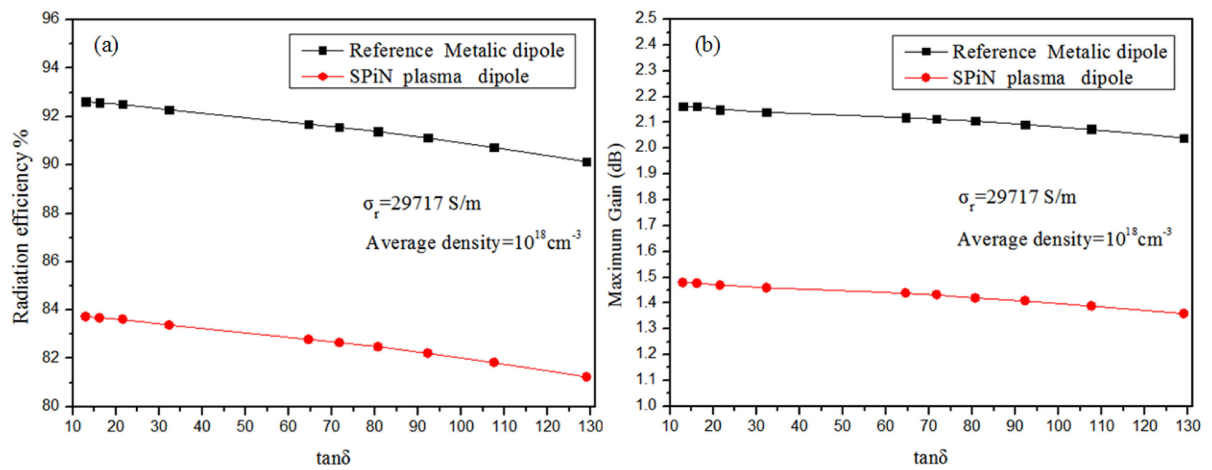

Fig. 9. The characteristics of the antenna

It can be seen from the Table I that the loss tangent $\tan \delta$ as a function of the imaginary part of the electrical conductivity $\left(\sigma_{\mathrm{i}}\right)$ and electromagnetic signal frequency (f) is decreasing with the increasing $\sigma_{\mathrm{i}}$ and f. The Fig. 9(a) and Fig. 9(b) show that the radiation efficiency and maximum gain of the antenna is better if the loss $\operatorname{tangent} \tan \delta$ is lower. And if the dipole antenna is made of metal, the radiation efficiency is $92.63 \%$ and maximum gain $2.16 \mathrm{~dB}$; if it was made out of a highly doped silicon SPiN array with average plasma concentration $\mathrm{n}=10^{18} \mathrm{~cm}^{-3}$ will lead to an efficiency $83.3 \%$ and maximum gain $1.48 \mathrm{~dB}$. Therefore, solid state plasma antenna appears to be a good candidate for the metal antenna.

\section{Conclusions}

A two dimensional electrical conductivity distribution model was proposed in this paper. The distribution of the real and imaginary part of electrical conductivity as a linear function of concentration is nonuniform in the I-region. And the imaginary part is more sensitive to the electromagnetic signal frequency than the real part, which will increase with the increasing electromagnetic signal frequency. Finally, the effect of the imaginary part on the plasma antenna has been studied by the loss $\operatorname{tangent} \tan \delta$ which is a function of imaginary part and signal frequency at a given average plasma concentration. Plasma dipole antenna has also been compared with metal dipole antenna, which make the plasma antenna instead of the metal antenna is possible. The proposed models can provide an effective reference for the design of the solid state plasma antenna.

\section{Acknowledgements}

This work has been supported by the National Natural Science Foundation of China (Grant No. 61474085). The authors would like to thank Professor Huiyong Hu for his many helpful discussions. 